\title{
Particle Induced X-ray Emission Imaging of Gadolinium Distribution into Xenograft U87 Human Glioblastoma after AGuIX Nanoparticles Injection
}

\author{
Asuncion Carmona ${ }^{1,2}$, Stephane Roudeau ${ }^{1,2}$, Richard Ortega ${ }^{1,2}$, Frederic Pouzoulet ${ }^{3}$ and Yolanda \\ Prezado ${ }^{4}$. \\ 1. CNRS, IN2P3, CENBG, UMR 5797, F-33170Gradignan, France. \\ 2. University of Bordeaux, CENBG, UMR 5797, F-33170Gradignan, France. \\ 3. Experimental radiotherapy platform, Institut Curie, 91405 Orsay, France. \\ 4. CNRS, IN2P3, IMNC, UMR 8165, 91405 Orsay, France.
}

In recent years, the use of high-Z nanoparticles (NPs) as potential tumor selective radiosensitizers has been proposed as a breakthrough in cancer radiotherapy (RT). NPs are capable of penetrating the cell and they lead to fewer adverse effects than conventional radiosensitizers [1]. Radiation sensitivity using NPs depends on cell line, irradiation energy, NPs type, size, concentration and distribution. High-Z metallic NPs ( $\mathrm{Au}, \mathrm{Gd}, \mathrm{Pt}, \mathrm{Ag}, \mathrm{Fe}$, etc.) have been predominantly used since they can generate shortrange photoelectrons or Auger electrons, which can enhance the therapeutic dose locally. It is of great interest to limit the diffusion of metallic NPs only to the tumor volume. So current investigations try to optimize the size of NPs and/or use chelating ligands of high affinity for tumor cells. Also the way that NPs are provided to the tumor, intravenously or by direct injection, will determine their distribution. Among those NPs, Gd-AGuIX (Activation and Guiding of Irradiation by X-ray) are $4 \mathrm{~nm}$ size polysiloxane GdNPs that have been proposed to be used for theragnostic as magnetic resonance imaging (MRI) contrast agents and radiotherapy sensitizers [2].

The purpose of our work was to determine the quantitative distribution of gadolinium into a xenograft U87 human glioblastoma tumor, after intratumoral injection of AGuIX NPs, using PIXE (Particle Induced X-ray Emission) imaging. PIXE is based on the detection of X-ray emitted by a sample when irradiated with a $\mathrm{MeV}$ ion beam, usually protons. This technique offers two important specific capabilities for trace metal analysis: imaging and quantification [3]. PIXE is a multi-elemental technique that allows determining elements with atomic number higher than 11 and that is more sensitive than electron microprobe. To study biological samples PIXE can be simultaneously carried out with RBS (Rutherford Backscattering Spectrometry) a technique which provide the local sample mass. This multimodal micro-spectrometry enables expressing metal content in terms of $\mu \mathrm{g}$ of element per $\mathrm{g}$ of dry mass all over the scanned area [4].

Female mices were grafted with human glioblastoma cells, U87, in right hind leg. $50 \mu \mathrm{L}$ of AGuIX NPs at $100 \mathrm{mM}$ were injected into the tumors and $1 \mathrm{~h}$ after, tumors were extracted and cryofixed. Tissue sections of $50 \mu \mathrm{m}$ thickness were deposited onto sample holders specially designed for PIXE analyses and tissue sections were freeze-dried [5]. Experiments were performed at AIFIRA facility (Applications Interdisciplinaires de Faisceaux d'Ions en Région Aquitaine) from CENBG laboratory (Centre d'Etudes Nucléaires de Bordeaux Gradignan). PIXE and RBS techniques were performed simultaneously to determine elemental content in tumor sections expressed in $\mu \mathrm{g}$ of element per $\mathrm{g}$ of dry mass. A proton beam was focalized at $2 \mu \mathrm{m}$ size and $500 \mathrm{pA}$ intensity. About 80 consecutive squared analyses of 750 $\mu \mathrm{m}$ side were performed to cover the all tumor slice area, of about $5 \mathrm{~mm}$ diameter (Fig. 1). Elemental images were reconstructed to overlay all scanned regions. 
We found that gadolinium was not homogeneously distributed into the tumor (Fig. 1). Gd appears in greater amount near the injection point, where content can reach $8000 \mu \mathrm{g} / \mathrm{g}$ dry mass, and decreases down to $100 \mu \mathrm{g} / \mathrm{g}$ within a few microns. Potassium distribution shows some regions with strong signal, which could correspond to high tumor cell density while $\mathrm{Gd}$ is not well incorporated within these areas. Iron distribution is not homogeneous, but in a spot shape and in the surrounding of the tumor, probably into the blood vessels. Silicium, from the polysiloxane structure, shows the same pattern distribution that Gd because both are ligands of AGuIX NPs. These results mean that care must be taken when interpreting MRI images because the tumor is not limited to the strong signal area. This distribution should also be taken into account in radiotherapy when tumor deposited doses are calculated. Overall, this study suggests that protocols to optimize the intratumoral distribution of NPs should be further investigated and that PIXE imaging can provide valuable information in this field.

\section{References:}

[1] JF Hainfeld, DN Slatkin and HM Smilowitz, Physics in Medicine and Biology 49 (2004), p. N309. [2] G Le Duc, S Roux, A Paruta-Tuarez, S Dufort, E Brauer, A Marais, C Truillet, L Sancey, P Perriat, F Lux and O Tillement, Cancer Nanotechnology 5 (2014), p. 4.

[3] R Ortega, G Deves and A Carmona, Journal of the Royal Society Interface 6 (2009), p. S649.

[4] A Carmona, G Deves and R Ortega, Analytical and Bionalytical Chemistry 390 (2008), p. 1585.

[5] L Perrin, A Carmona, S Roudeau and R Ortega, Journal of Analytical Atomic Spectrometry 30 (2015), p. 2525.

[6] The authors acknowledge Baptiste L'homel for his help during data treatment.

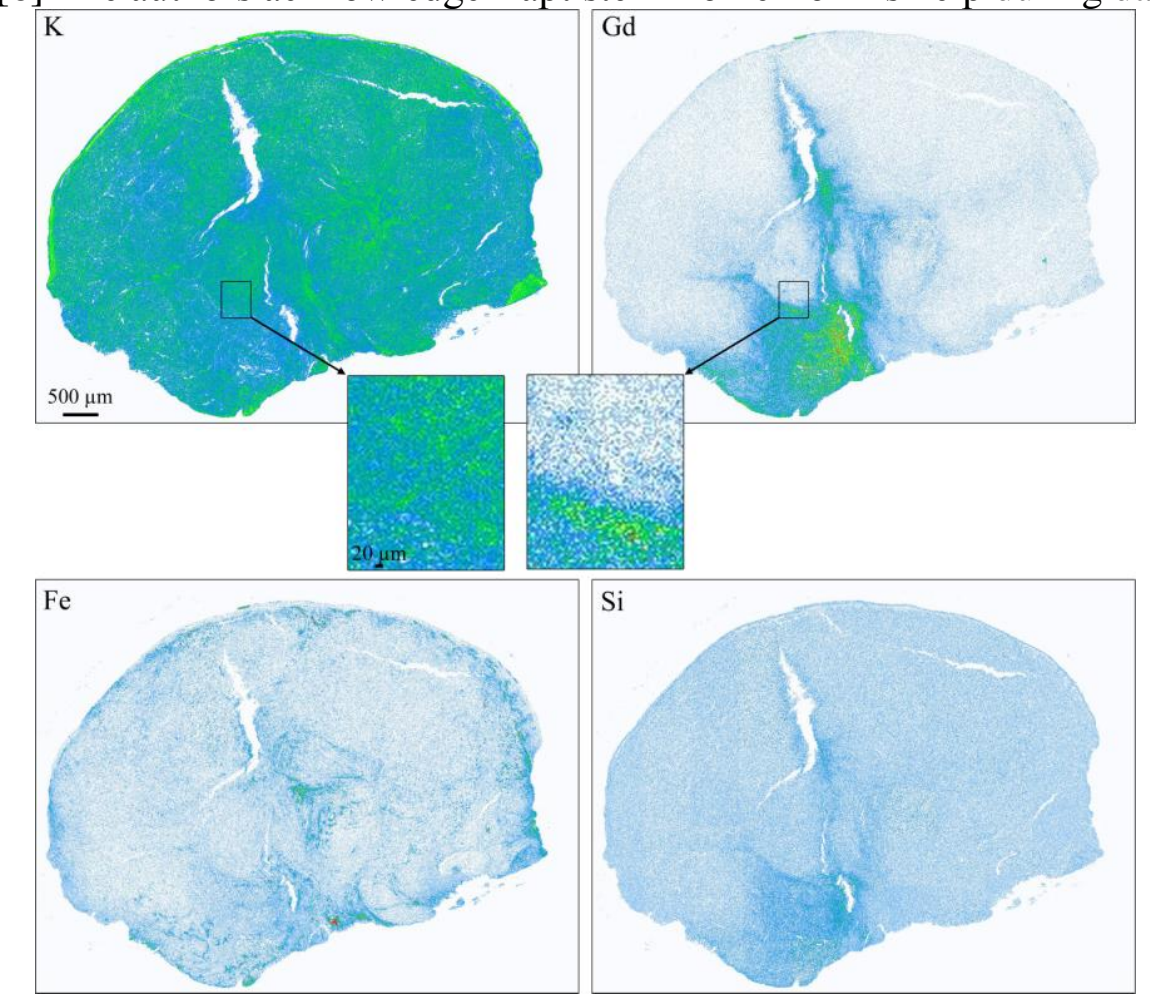

Figure 1. Elemental distribution of $\mathrm{K}, \mathrm{Fe}, \mathrm{Gd}$ and $\mathrm{Si}$ into a thin section of xenograft U87 human glioblastoma tumor, injected with AGuIX NPs. Zoom showing that Gd and K are not co-localized. Gd is not homogeneously distributed into the tumor and accumulates near the injection point. 